\title{
The use of polypropylene mesh in inguinal hernia surgery: A retrospective study
}

\author{
CIPRIAN TANASESCU ${ }^{1,2}$, ANDREI MOISIN ${ }^{1}$, ALIN MIHETIU $^{1,2}$, DRAGOS SERBAN $^{3}$, \\ ADRIAN COSTACHE ${ }^{4}$ and DAN GEORGIAN BRATU ${ }^{1,2}$ \\ ${ }^{1}$ Department of Surgery, Sibiu County Clinical Emergency Hospital, 550245 Sibiu; \\ ${ }^{2}$ Clinic Surgical Department, Faculty of Medicine, 'Lucian Blaga' University, 550169 Sibiu; \\ ${ }^{3}$ The 4th Surgery Department, Emergency University Hospital, 050098 Bucharest; \\ ${ }^{4}$ Department of Pathology, 'Carol Davila' University of Medicine and Pharmacy, 020021 Bucharest, Romania
}

Received May 21, 2021; Accepted June 22, 2021

DOI: $10.3892 /$ etm.2021.10627

\begin{abstract}
Previously, inguinal hernia surgery was based exclusively on repairing the abdominal wall defects using the patient's own tissues, which were put in contact with and tensioned to recalibrate the natural orifices. At present, inguinal hernia surgery is based almost solely on mounting an allograft, which has the role of strengthening the weakened groin region that allowed the herniation. This modern method of operation on inguinal hernia can be performed in a classic or laparoscopic manner. The mesh is made of polypropylene, which is a polymer of cyclic hydrocarbons. The aim of the present study was to evaluate the effectiveness, biocompatibility, as well as the immediate and long-term complications in textile allografts used in open surgery of inguinal hernia repair. Another aim was to demonstrate once again the superiority of low-weight meshes with large pores by decreasing the number of complications caused by the synthetic material used, but also by a decrease in the tension on the tissues to which it was fixed. The present study included 255 cases submitted to inguinal hernia surgery. Only $1.5 \%$ required immediate reintervention before discharge to evacuate hematoma. The short duration of hospitalization, the quality-price ratio, the good postoperative results, as well as the rapid socio-professional reintegration, render the use of polypropylene mesh in inguinal hernia surgery very attractive for patients.
\end{abstract}

\section{Introduction}

Inguinal hernia represents the entrance of peritoneal viscera through the inguinal canal. The viscera are enveloped by

Correspondence to: Dr Adrian Costache, Department of Pathology, 'Carol Davila' University of Medicine and Pharmacy, 37 Dionisie Lupu Street, 020021 Bucharest, Romania

E-mail: adriancostacheeco@yahoo.com

Key words: polypropylene mesh, inguinal hernia, allografts, complications, one-day surgery the peritoneum forming the hernial sac. The lifetime risk to develop an inguinal hernia is $27-43 \%$ for men and $3-6 \%$ for women. Surgical closure of the hernia sac has become of the most commonly used procedure in general surgery (1).

The surgical treatment of inguinal hernia can be performed using a variety of techniques, all based on the treatment of the hernia sac and its contents, but also the restoration of the abdominal wall. In the XV-XVI centuries, empirical methods were used, which were abandoned in the XVIII century, when the first manuscripts appeared containing information related to the treatment of hernia (2). The 19th century marks the beginning of the modern era of hernia surgery with the appearance of the Cooper and Scarpa manuscripts (3). In 1984, E. Bassini introduced the idea that the weakness of the posterior wall of the inguinal canal is responsible for the production of a hernia (4). As a result, he introduced the idea of strengthening the posterior wall by suturing the lower edge of the deep abdominal muscles to the femoral arch. For this reason, the great surgeon Halsted considered Bassini, 'the father of hernia surgery' (5). The revolution in the treatment of hernias began with the use of the principle of 'Tension-Free hernioplasty', in which the tension in the structures of the inguinal canal disappears, an idea promoted by French surgeons J. Rives and R. Stoppa (6). They used a polymer mesh for the first time in the repair of the hernia, placed between the peritoneum and the transversalis fascia. In 1993, Lichtenstein published the results of 3,125 hernioplasties, in which he used a polypropylene mesh placed above the transversalis fascia, a study in which only 4 cases recurred (7).

There are various repair techniques for inguinal and femoral hernias, classified as classical wide open approaches or laparoscopic techniques such as: The total extraperitoneal patch plasty (TEP), transabdominal preperitoneal patch plasty (TAPP), and Lichtenstein techniques $(1,8)$. The concept of using a mesh to repair hernias was introduced over 50 years ago and is now standard in most countries and widely accepted as superior to primary suture repair. As a result, there has been a rapid growth in the variety of meshes available and choosing the appropriate one can be an extremely difficult task (9).

The aim of the present study was the evaluation of the effectiveness of allograft mesh, as well as the immediate and 
long-term complications in textile allografts used in open surgery of inguinal hernia repair.

\section{Materials and methods}

Ethics approval and patient consent. The present study followed the international regulations in accordance with the Declaration of Helsinki. The study was approved by the Ethics Committee of the Sibiu County Clinical Emergency Hospital. Patient informed consent for publication of the data/images associated with the manuscript was obtained.

Materials. The current study includes cases admitted to the Department of Surgery of Sibiu County Clinical Emergency Hospital for the period January 2011 to December 2018. The study included 255 patients over a 7-year period, who underwent the modified Lichtenstein procedure using Premiline Mesh $^{\mathrm{TM}}$. Patients from January 2019 to December 2020 were not included due to the Covid-19 pandemic and potential statistical bias. The mesh was constructed from monofilament polypropylene, which was knitted into a thin and elastic shape-stable mesh with large pores and low weight (LW). Statistical analysis was performed using Excel Suite Software.

This type of allograft was selected as it is part of the LW and large-pore mesh group. The reasons for the choice were: Easier handling, reduced contact with tissues due to the large pores of the mesh (which leads to a decrease in the number of complications related to net rejection, seromas, granulomas) and the numerous studies performed on swine models whose results have demonstrated their increased efficacy $(10,11)$.

The meshes used had thinner threads and pores $>1 \mathrm{~mm}$. Their specific weight was $33 \mathrm{~g} / \mathrm{m}^{2}$. These meshes have less tissue reaction and are more elastic in behavior. The elasticity of all LW meshes ranges from 20 to $35 \%$ at $16 \mathrm{~N} / \mathrm{cm}$. Modern biomaterials have to be both physical and chemical inert as the tissue reaction occurs in close relationship with the diameter of the pores and the amount of foreign material inserted into the body (12).

Surgical technique description. The technique used in all cases was the Lichtenstein procedure, a well-known procedure that is not described herein (13). However, particular aspects that led to modifying the classical technique include the following.

i) The anesthesia used was a combination of ilioinguinal block, local and intravenous anesthesia. The ilioinguinal block was calculated as follows: $2 \mathrm{~cm}$ were measured laterally from the iliac spine on the side of the hernia on the bispinous line, then $2 \mathrm{~cm}$ cranially on the perpendicular drawn on the bispinous line, at the point located at $2 \mathrm{~cm}$ (Fig. 1). Local anesthesia was performed by skin infiltration at the incision site. For intravenous anesthesia, a general anesthetic from the class of non-barbiturates was used.

ii) This type of anesthesia associated with the concept of Fast Track Surgery (FTS) is indispensable in one-day surgeries. The concept of FTS involves a combination of techniques used in order to provide a very fast recovery after surgery. It includes elements of anesthesia, surgery, nutrition, and rapid postoperative mobilization.

iii) The incision was transverse, parallel to the bispinous line, but centered on the inguinal canal at half the distance between the superficial and the deep orifice (Fig. 2). iv) The allograft used was of the Premiline Mesh type, with large pores and low weight, which we adapted to the anatomy of the specific region of each patient.

v) At the end of the intervention, the patient is woken up on the operating table and helped to walk on their feet to the postoperative or recovery room-respecting the same concept of FTS.

vi) The discharge was made $2-3 \mathrm{~h}$ after the end of the surgery (7).

Hematoxylin and eosin staining. To identify any subcutaneous surgical thread granulomas, tissues were assessed using a conventional brightfield microscopy. The samples were fixed with $10 \%$ formaldehyde, at room temperature for 24 h. The tissue fragments were dehydrated and submitted to 3 toluene passages prior to paraffin inclusion. The sections were 3 microns thick. Hematoxylin and eosin staining was carried out at room temperature for $1 \mathrm{~h}$. Giant cells were identified under an optical microscope Viola MC20i microscope using an SP ARCHO lens.

Statistical analysis. Collected data were compiled and tabulated on a master sheet. The results obtained were subjected to basic statistical analysis in Microsoft Excel 16.45 (Microsoft Corporation). Besides descriptive statistics (mean \pm standard deviation), bivariate analysis was performed using Pearson's Chi-square and Fisher's exact test for categorical variables as applicable, and the Student's t-test for continuous variables. $\mathrm{P}<0.05$ was considered to indicate statistically significant results.

\section{Results and Discussion}

Distribution of patients according to age. Of the total number of patients included in the study group, 206 were male and 49 were female. The 255 patients in this study had a male to female ratio of $81 \%$ males and $19 \%$ females, this being in total accordance with the worldwide distribution for this type of surgical pathology. The patients' age ranged between 20 and 90 years. Regarding the distribution of patients by age groups, 74 patients were in the 20-40 age group, 85 patients in the 41-61 age group and 96 patients in the 61-90 age group $(\mathrm{P}=0.09)$ (Table I).

Out of the 255 cases, 51 patients $(20 \%)$ resided in a rural environment, while the remaining 204 patients (80\%) resided in an urban environment $(\mathrm{P}=0.006)$. This aspect is very important because untreated minor early complications may lead to subsequent major problems. Of note, in Romania the residence of the patient greatly influences the quality of postoperative care and follow up due to a decreased accessibility to healthcare services in rural areas. Needless to say disease prevention is decreased, and the patients tend to postpone the initial consult and present with advanced pathology stages.

Distribution of patients with postoperative complications. Postoperative evaluation of the patients is essential in monitoring both immediate and late complications, as well as in evaluating the prosthesis. The monitoring of the patients who were discharged was performed at 2 and 7 days. In addition, all patients were monitored at 1 and 6 months by performing an abdominal ultrasound. 


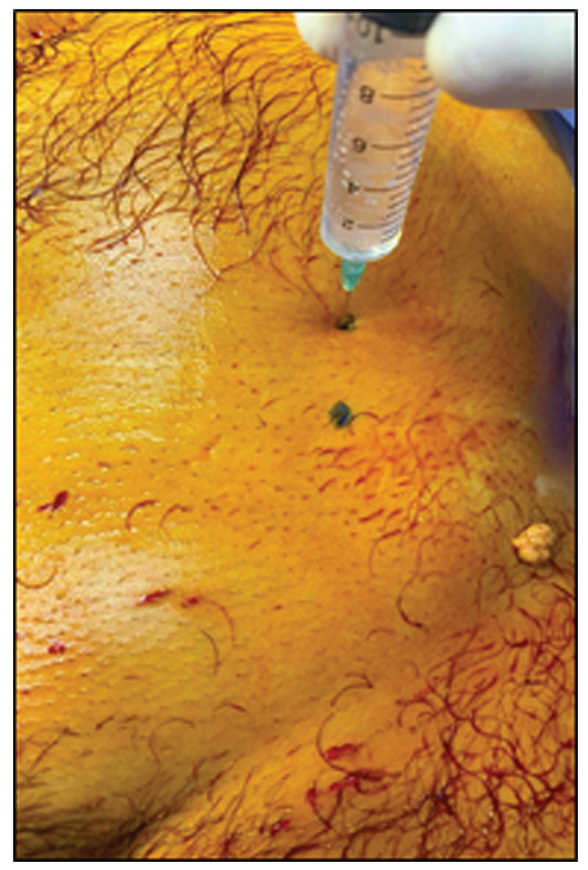

Figure 1. Ilioinguinal nerve block for local regional anesthesia.

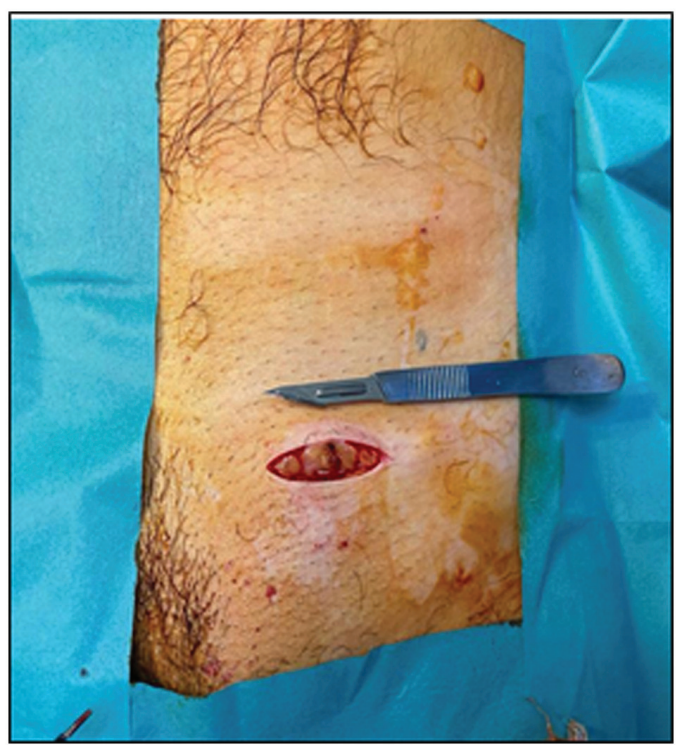

Figure 2. The 5-cm incision for minimal approach.

At the 2-day check-up, there were only 4 cases (1.5\%) of large postoperative hematoma that required surgical reintervention, although without removal of the allograft. The remaining 251 patients included in the study group did not present immediate complications at the first postoperative evaluation $(\mathrm{P}=0.0033)$ (Table II).

At the 7-day check-up, on which occasion the sutures were suppressed, complications were observed in 25 patients, distributed as follows: In 9 patients there were hematomas and in 16 seromas $(\mathrm{P}=0.04)$ (Table II).

In addition, there were no superinfections of the postoperative wound, probably due to the prophylaxis performed in each case at the anesthetic induction by intravenous administration of $1 \mathrm{~g}$ amoxicillin.
Table I. Distribution of patients according to age.

\begin{tabular}{lcc}
\hline Age group & No. of cases & Percentage \\
\hline $20-40$ & 74 & 29.1 \\
$41-61$ & 85 & 33.3 \\
$61-90$ & 96 & 37.6 \\
Total & 255 & 100 \\
\hline
\end{tabular}

Table II. Distribution of patients with early postoperative complications.

\begin{tabular}{lccc}
\hline & \multicolumn{2}{c}{ Immediate postoperative complications } \\
\cline { 2 - 4 } Check-up & $\begin{array}{c}\text { Large } \\
\text { hematoma }\end{array}$ & $\begin{array}{c}\text { Small } \\
\text { hematoma }\end{array}$ & $\begin{array}{c}\text { Wound } \\
\text { seroma }\end{array}$ \\
\hline 2 days & 4 & - & - \\
7 days & - & 9 & 16 \\
Total & 4 & 9 & 16 \\
\hline
\end{tabular}

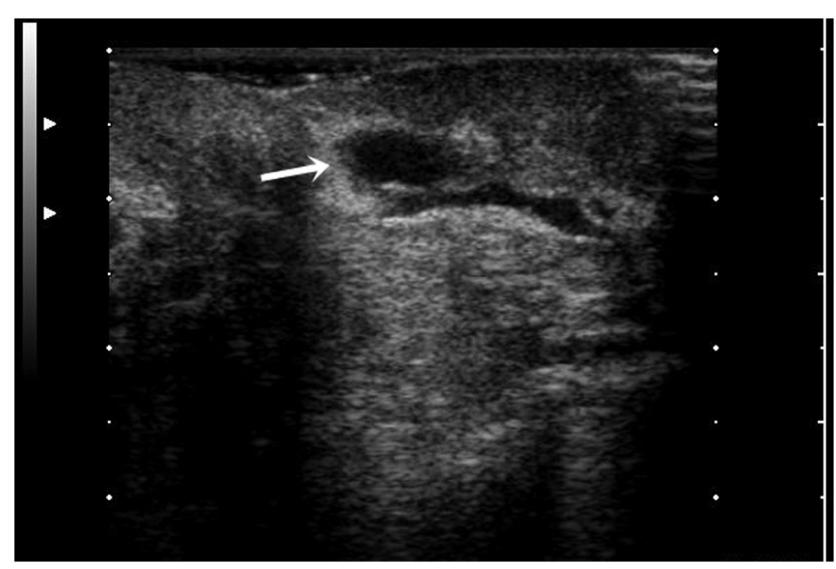

Figure 3. Seroma identified above the mesh. Ultrasound image one month after the surgery.

Late postoperative morbidity was represented by chronic postoperative pain in 6 patients and cutaneous hypoesthesia in 14 patients. All 25 cases had a spontaneous resolution.

Regarding ultrasound monitoring one month after discharge, 38 patients developed seroma, 25 patients developed subcutaneous surgical thread granulomas as viewed under a Viola MC20i microscope using an SP ARCHO lens, 13 patients developed granulomatous subcutaneous fibrotic band and 8 of them developed granulomatous over the mesh fibrotic band (Figs. 3 and 4$)(\mathrm{P}=0.01)$.

These 84 patients also presented late complications in the first month, detected using abdominal ultrasound, representing $33 \%$ of the total number of patients included in the study group $(\mathrm{P}=0.011)$.

In a significant number of cases from the present study, tissue reactivity was identified in the form of granulomas, seromas or lymph-node reaction. This reactivity can be explained by the materials used and tissue properties, surgical 


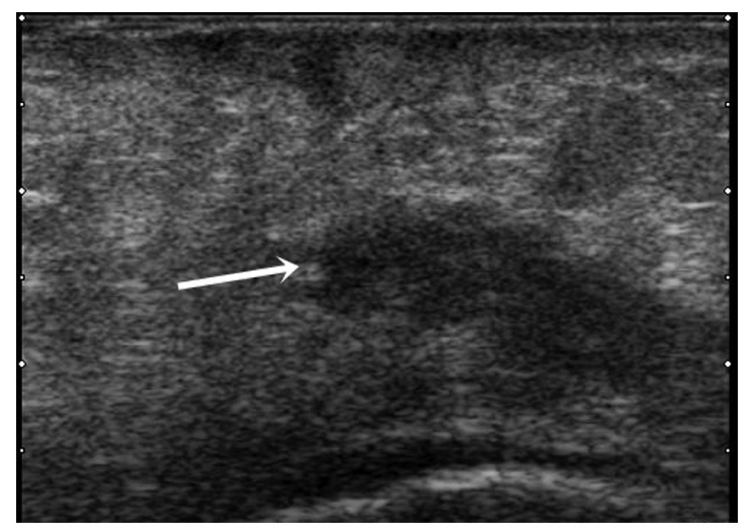

Figure 4. Postoperative granuloma identified above the mesh. Ultrasound image at one month follow-up.

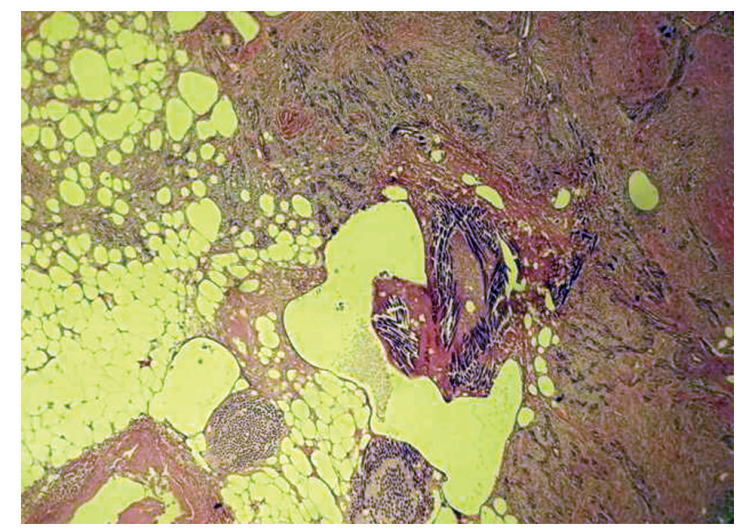

Figure 5. Surgical thread granuloma. Giant cells of inflammatory reaction with thread highlighting. Viewed under a Viola MC20i microscope using an SP ARCHO lens (magnification, $\mathrm{x} 4$ ); hematoxylin and eosin staining.

technique, as well as by the immune/inflammatory response of each patient. The body is considered a key factor regarding a complete integration of the mesh at the tissue level.

Foreign body granuloma occurs through a non-immune mechanism. It is triggered by exogenous (in our case mesh) or endogenous foreign bodies. Microscopically, the foreign body granuloma in the case of the mesh is made up of giant multinucleated cells, with a disordered nucleus, resulting from the fusion of macrophages that come to phagocytose the foreign body. The foreign body is bi-refractive, located in the center of the granuloma, without necrosis (Figs. 5 and 6).

Patient follow-up. The patients who had immediate postoperative complications were called for a new check-up at 6 months postoperatively. Furthermore, a soft tissue ultrasound was performed, which showed the disappearance of the seromas, but in 6 patients, the persistence of inguinal lymphadenopathy was found (Figs. 7 and 8$)(\mathrm{P}=0.02)$. Another important point in the ultrasound evaluation that was performed at 6 months was the study of how the mesh was accepted by the host organism. Thus, in only $30 \%$ of cases, the allograft was visible on ultrasound examination, while in the remaining patients it could no longer be highlighted $(\mathrm{P}=0.009)$.

A high percentage of small-sized postoperative seromas was observed. This could be due to enzymatic activity and

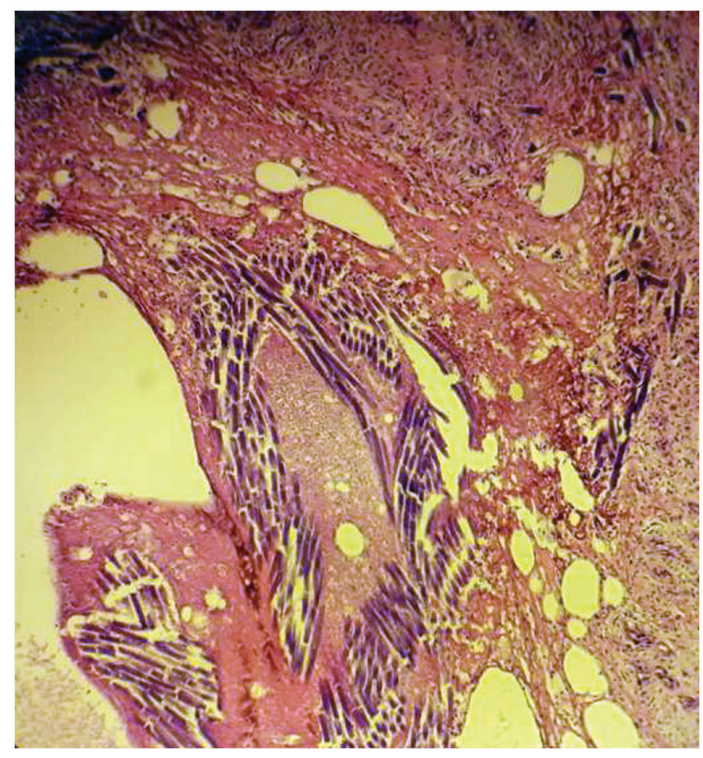

Figure 6. Surgical thread granuloma. Giant cells of inflammatory reaction with thread highlighting. Viewed under a Viola MC20i microscope using an SP ARCHO lens (magnification, x10); hematoxylin and eosin staining.

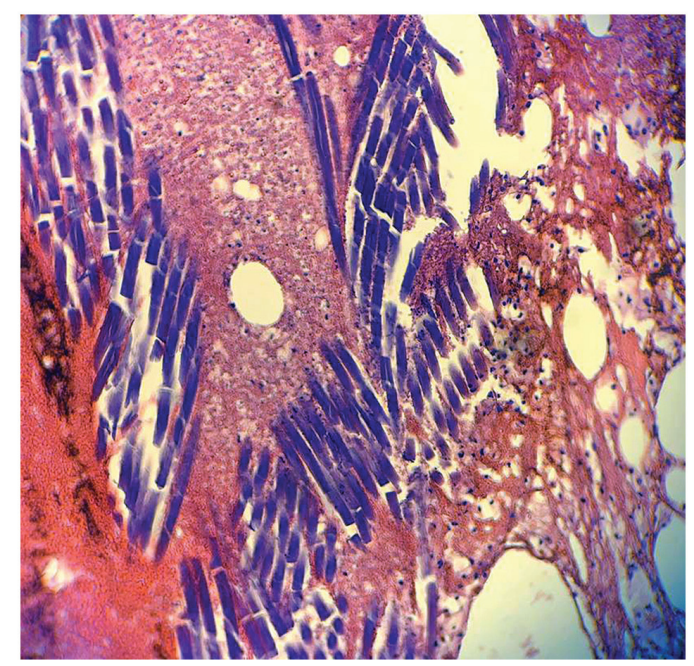

Figure 7. Surgical thread granuloma. Giant cells of inflammatory reaction with thread highlighting. Viewed under a Viola MC20i microscope using an SP ARCHO lens (magnification, x40); hematoxylin and eosin staining.

tissue reactivation that is overrun in the immediate postoperative period. In their composition, lymph, proteins, blood (small amounts), fibrin, LDH, and white blood cells (WBC) were found. The presence of WBC with neutrophilia at higher values locally in the blood stream clearly indicates local tissue activity (14).

The mesh with large pores and low weight was employed as there was low contact between the allograft and the body's own tissues, which led to improved acceptance of the alloplastic material by the host organism. The presence of small holes in the mesh $(<1 \mathrm{~mm})$ pronounced the inflammatory process and fibrosis, while larger holes provided less fibrotic reaction, and conserved the elasticity of the inguinal region and lack of keloid scar (15-17).

Alloplastic mesh with large holes was used exclusively in the current study. The reasons behind this decision were the 


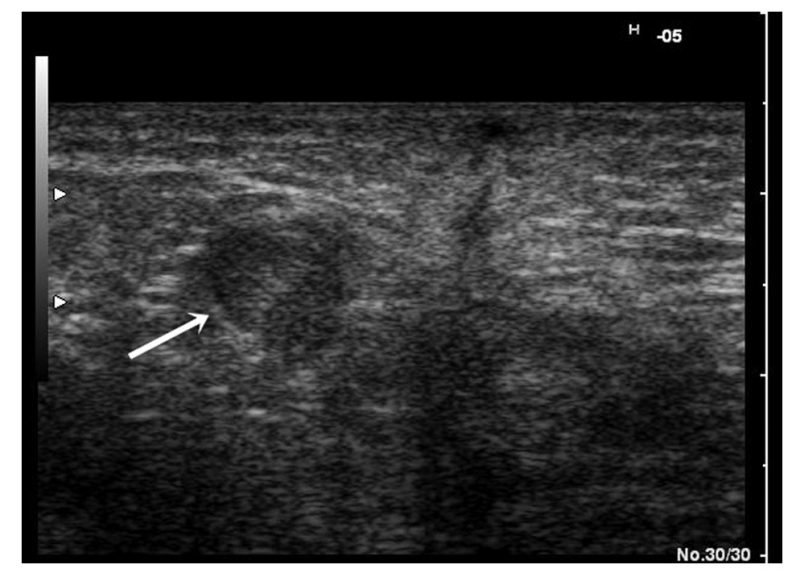

Figure 8. Inguinal adenopathy. Ultrasound image at 6 months postoperative follow-up.

advantages of large-pore LW meshes. Larger pore size leads to improved integration and biochemical capacity, as well as increased tissue ingrowth. This type of mesh has a better integration rate compared to LW meshes with small pores and the patient is less conscious of the foreign body. A major disadvantage of this type of mesh is the lack of stability that leads to shrinkage. The LW small pore mesh was not utilizes as it has a significantly higher shrinkage rate than any other types of mesh and also a lower tissue integration rate. Numerous clinical studies underlined the important of a tension-free placement of the mesh (18-21).

Effect of mesh. A common complication of the procedures when dissecting extensively the hernia sac is the development of seromas. A seroma hinders the attachment of the patch to the surrounding tissues with rapid fixation and increases the risks of recurrence and infection (22).

Among the factors leading to seroma formation are senescence, large hernia volume and scrotum involvement. However, a recent meta-analysis did not note any difference in the impact of lightweight vs. heavyweight meshes on the seroma rate after inguinal hernia repair (23).

Another frequent complication is the development of hematoma. The risk of hematoma is increased by partially absorbable mesh, chronic anticoagulation, recurrent hernia procedure, mesh fixation, larger hernia defect and medial defect localization (24).

Regarding the formation of hematomas and seromas, their occurrence is more related to the application of the technique and the risk factors of each patient than the technique itself (7).

Needless to say, all 255 cases underwent a complex differential diagnosis for other possible associated pathology such as gastro-intestinal carcinomas (25). Other pathology influencing surgical recovery is diabetes and viral coinfections (26). The fact that we did not encounter a case with complete immunologic rejection of the mesh is encouraging (27).

The approach of this type of pathology in a welded medical-surgical team, with a constant number of cases operated monthly, leads to decreased operative time, decreased number of intraoperative and postoperative complications and with improved immediate and distant results. Using the FTS concept, together with the use of prostheses, helps increase the patient's chances for a rapid socio-professional reintegration, with minimal immediate and late postoperative complications.

Currently, the use of polypropylene mesh represents the best option to restore the integrity of the abdominal wall in patients diagnosed with inguinal hernia. In addition, the use of polypropylene allografts with wide holes has contributed to a significant reduction of the local inflammatory process, as indicated in aforementioned literature. Polypropylene has proven physical, chemical and biological properties, and is currently the most widely used allograft in the treatment of inguinal hernias.

The local inflammatory response produced by the contact between the tissues and the mesh causes a delay in the complete integration of the tissues, but it is not associated with the immediate recurrence of the herniated sac and the development of complications is limited.

Using LW polypropylene allograft with thinner threads and large pores improved abdominal wall compliance, with less contraction or shrinkage of the mesh and allowed improved tissue incorporation.

During the 7-year duration of the study, there were no cases of recurrence of the hernia sac. This is due both to the extensive experience in the medical centers included in the study and to the quality materials used.

Ilioinguinal block anesthesia, together with intravenous anesthesia, is an important factor in the rapid recovery of patients. The short duration of hospitalization $(<4 \mathrm{~h})$, the quality-price ratio, the good postoperative results, as well as the rapid socio-professional reintegration, render the technique very attractive for patients.

The multimodal approach of the patients diagnosed with inguinal hernia using a series of preoperative workouts together with the combined anesthesia, the adaptation of the Lichtenstein technique and a quality mesh, constitutes a step forward in the modern treatment of inguinal hernia.

\section{Conclusion}

Findings of the present study demonstrate once again the conclusions of literature according to which LW meshes with large pores are superior to those with small pores, due to an optimized foreign body reaction based on small amounts of mesh and, in particular, a significantly low surface area in contact with the host tissues through the large porous pattern. In addition, an extremely important feature of this type of mesh is the progressive decrease of shrinkage on the tissues to which they are fixed, decreasing the risk of recurrence. In the present study, there was no case of recurrence at the 6-month check-up. Postoperative complications related to mesh quality were extremely low, similar to those in international studies.

\section{Acknowledgements}

Professional editing, linguistic and technical assistance were performed by Irina Radu, Individual Service Provider.

\section{Funding}

No funding was received. 


\section{Availability of data and materials}

All data generated or analyzed during this study are included in this published article.

\section{Authors' contributions}

CT and AMo contributed substantially to the conception and design of the study, the acquisition, analysis, and interpretation of the data, and were involved in the drafting of the manuscript. AMi and DS contributed substantially to the analysis and interpretation of the data and were involved in the drafting of the manuscript. AC and DGB contributed substantially to the interpretation of the data and were involved in the critical revisions of the manuscript for important intellectual content. CT, AMo and DS are responsible for confirming the authenticity of all the raw data. All authors agreed to be accountable for all aspects of the work in ensuring that questions related to the accuracy or integrity of any part of the work are appropriately investigated and resolved. All authors read and approved the final version of the manuscript.

\section{Ethics approval and consent to participate}

The study followed the international regulations in accordance with the Declaration of Helsinki. The study was approved by the Ethics Committee of the Sibiu County Clinical Emergency Hospital (approval no. 20200518). Patient informed consent for publication of the data/images associated with the manuscript was obtained.

\section{Patient consent for publication}

Patient informed consent for publication of the manuscript was obtained.

\section{Competing interests}

The authors declare that they have no competing interests.

\section{References}

1. Köckerling F and Simons MP: Current concepts of inguinal hernia repair. Visc Med 34: 145-150, 2018.

2. White JJ and Haller JA: Groin Hernia in Infants and Children. Nyhus LM and Condon RE (eds). Hernia Philadelphia, PA, Lippincott, pp101-136, 1978.

3. Rutkow MI: A selective history of groin hernia surgery in the early 19th century: The Anatomic Atlases of Astley Cooper, Franz Hesselbach, Antonio Scarpa, and Jules-Germain Cloquet. Surg Clin North Am 78: 921-940, 1998.

4. Dørflinger T and Kiil J: Absorbable suture in hernia repair. Acta Chir Scand 150: 41-43, 1984.

5. Summers JE: Classical herniorrhaphies of Bassini, Halsted and Ferguson. Am J Surg 73: 87-99, 1947.

6. Stoppa R, Petit J, Abourachid H, Henry X, Duclaye C, Monchaux G and Hillebrant JP: Original procedure of groin hernia repair: Interposition without fixation of Dacron tulle prosthesis by subperitoneal median approach. Chirurgie 99: 119-123, 1973 (In French).

7. Tanasescu C, Faur M and Sabau D: Day-case surgery in the context of inguinal hernia repair by the modified Lichtenstein technique-A single Centre experience. Chirurgia (Bucur) 114: 115-120, 2019.

8. Hernia Surge Group: International guidelines for groin hernia management. Hernia 22: 1-165, 2018.
9. Brown CN and Finch JG: Which mesh for hernia repair? Ann R Coll Surg Engl 92: 272-278, 2010.

10. Bratu D, Boicean A, Tanasescu C, Sofariu C, Mihetiu A, Mitariu Cernusca IS, Ognean L, Moldovan C and Boitor C: Textile polypropylene allografts and their postoperative tissue reaction in the surgery of inguinal hernia. Mater Plast 54: 119-121, 2017.

11. Cobb WS, Burns JM, Peindl RD, Carbonell AM, Matthews BD, Kercher KW and Heniford BT: Textile analysis of heavy weight, mid-weight, and light weight polypropylene mesh in a porcine ventral hernia model. J Surg Res 136: 1-7, 2006.

12. Bolocan A, Ion D, Constantinescu S, Luca AD and Paduraru DN: Randomised trial comparing poly-propylene mesh and polyvinylidene fluoride mesh in the surgery. Mat Plast 49: 209-211, 2012.

13. Lichtenstein IL, Shulman AG, Amid PK and Montllor MM: The tension-free hernioplasty. Am J Surg 157: 188-193, 1989.

14. Wotton FT and Akoh JA: Rejection of Permacol mesh used in abdominal wall repair: A case report. World J Gastroenterol 15: 4331-433, 2009.

15. Kehlet H and Bay Nielsen M: Anaesthetic practice for groin hernia repair-a nation-wide study in Denmark 1998-2003. Acta Anaesthesiol Scand 49: 143-146, 2005.

16. Fitzgibbons RJ Jr, Giobbie-Hurder A, Gibbs JO, Dunlop DD, Reda DJ, McCarthy M Jr, Neumayer LA, Barkun JST, Hoehn JL, Murphy JT, et al: Watchful waiting vs. re-pair of inguinal hernia in minimally symptomatic men: A randomised clinical trial. JAMA 295: 285-292, 2006.

17. Chicea R, Chicea AL, Mihetiu A, Tantar C, Cernusca Mitaru MM, Cernusca Mitaru SI, Roman C, Comaneanu RM, Bratu D and Manea MM: Comparative study between tissues induced immunohistochemical changes of thread granulomas and textile allografts. Mater Plast 55: 99-101, 2018.

18. Weyhe D, Cobb W, Lecuivre J, Alves A, Ladet S, Lomanto D and Bayon Y: Large pore size and controlled mesh elongation are relevant predictors for mesh integration quality and low shrinkage-Systematic analysis of key parameters of meshes in a novel minipig hernia model. Int J Surg 22: 46-53, 2015.

19. Klosterhalfen B, Junge $K$ and Klinge U: The lightweight and large porous mesh concept for hernia repair. Expert Rev Med Devices 2: 103-117, 2005.

20. Wolak PK, Strzelecka A, Piotrowska A, Dąbrowska K, Wolak PP, Piotrowska I and Nowak-Starz G: The operative time for unilateral inguinal hernia repair in children performed with Percutaneous Internal Ring Suturing (PIRS) or open approach method. J Clin Med 10: 1293, 2021.

21. Sanbhal N, Miao L, Xu R, Khatri A and Wang L: Physical structure and mechanical properties of knitted hernia mesh materials: A review. J Industrial Textiles 48: 333-360, 2018.

22. Fang H, Lin R, Lin X, Lu F, Yang Y, Wang C, Chen Y and Huang H: Drainage decreases the seroma incidence in laparoscopic transabdominal preperitoneal (TAPP) hernia repair for large inguinoscrotal hernias. Asian J Surg 44: 544-548, 2021.

23. Köckerling F, Bittner R, Adolf D, Fortelny R, Niebuhr H, Mayer F and Schug-Pass C: Seroma following transabdominal preperitoneal patch plasty (TAPP): Incidence, risk factors, and preventive measures. Surg Endosc 32: 2222-2231, 2018.

24. Smoot RL, Oderich GS, Taner CB, Greenlee SM, Larson DR, Cragun EB and Farley DR: Postoperative hematoma following inguinal herniorrhaphy: Patient characteristics leading to increased risk. Hernia 12: 261-265, 2008.

25. Georgescu EF, Mogoantă SŞ, Costache A, Pârvănescu V, Totolici BD, Pătraşcu Ș and Stănescu C: The assessment of matrix metalloproteinase-9 expression and angiogenesis in colorectal cancer. Rom J Morphol Embryol 56: 1137-1144, 2015.

26. Rusu E, Jinga M, Rusu F, Ciurtin C, Enache G, Dragomir A, Cristescu V, Stoica V, Costache A, Cheta D and Radulian G: Statin therapy in patients with diabetes and hepatitis C. Farmacia 61: 1204-1215, 2013

27. Berghi NO, Dumitru M, Vrinceanu D, Ciuluvica RC, Simioniuc-Petrescu A, Caragheorgheopol R, Tucureanu C, Cornateanu RS and Giurcaneanu C: Relationship between chemokines and $\mathrm{T}$ lymphocytes in the context of respiratory allergies (Review). Exp Ther Med 20: 2352-2360, 2020.

This work is licensed under a Creative Commons Attribution-NonCommercial-NoDerivatives 4.0 International (CC BY-NC-ND 4.0) License. 